\title{
fulgeo-Towards an Intuitive User Interface for a Semantics-enabled Multimedia Search Engine
}

\author{
D. Schneider ${ }^{1}$, D. Stohr ${ }^{1}$, J. Tingvold ${ }^{2}$, A. B. Amundsen ${ }^{2}$, L. Weiland ${ }^{1}$, S. Kopf ${ }^{1}$, W. Effelsberg ${ }^{1}$, A. Scherp ${ }^{3}$ \\ ${ }^{1}$ University of Mannheim, Germany; ${ }^{2}$ University of Oslo, Norway; \\ ${ }^{3}$ Kiel University and Leibniz Information Center for Economics, Kiel, Germany
}

\begin{abstract}
Multimedia documents like PowerPoint presentations or Flash documents are widely adopted in the Internet and exist in context of lots of different topics. However, so far there is no user friendly way to explore and search for this content. The aim of this work is to address this issue by developing a new, easy-to-use user interface approach and prototype search engine. Our system is called fulgeo and specifically focuses on a suitable multimedia interface for visualizing the query results of semantically-enriched Flash documents.
\end{abstract}

\section{INTRODUCTION}

Flash content and multimedia content in general is quite popular and widely used today. Still, there exists no reasonable way to actually search for, find, and explore Flash documents on the Internet. Search engines like Google limit their support by presenting Flash presentations merely as textual results. Users can click on a result to view the presentation, but the engines provide no further intuitive ways for exploring the content. The speciality of multimedia content is that it consists of a collection of images, videos, sounds, and text assets [1]. These media assets are arranged in time and space. They can be animated or rearranged by user interactions, which affects the temporal and spatial rendering of the presentation. Due to these special features, it is not sufficient to provide only a text based search result page like Google, but to address all aspects of multimedia including the semantics that is captured in the entities of the text assets and the spatio-temporal information encoded in the arrangement of the assets [7]. To the best of our knowledge, FLAME (Flash Access and Management Environment) [8] is the only search engine for multimedia content today. The focus of FLAME is on the indexing and retrieval of Flash content. The search user interface consists of an input field for keyword-queries and the results are displayed in a grid like it is shown in Figure 1(a). A link to the details of a presentation is provided and a button to find similar flash files [3]. FLAME provides an "Advanced Search" page depicted in Figure 1(b) where users can enter more sophisticated queries via an input form such as shape, color, and media type. While such a query form represents the information that is actually encoded in the Flash presentations, we believe that a casual user will have significant difficulties filling out the form.

Thus, with fulgeo ("flash" in Latin) we propose a new and more intuitive user interface (UI) approach for searching semantically-enriched multimedia content. It is motivated from and based on earlier work on analysing a larger corpus of Flash documents obtained from the web [7]. We have

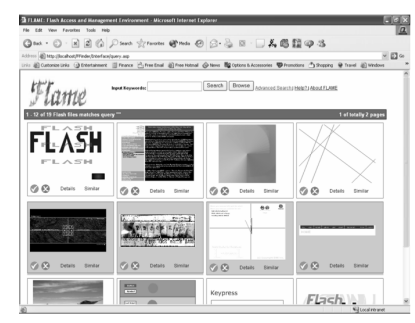

(a) Main UI

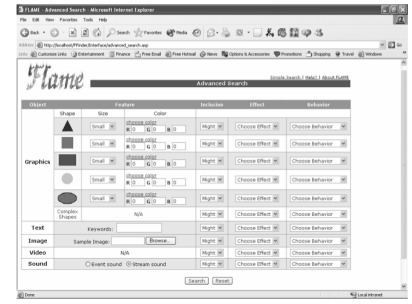

(b) Advanced UI
Fig. 1. FLAME search engine

extracted the media assets and the relation between the media assets from the Flash documents and represent them using a formal ontology. In addition, we connect the media assets with background knowledge on the web. To this end, we semantically enrich the text assets extracted from the multimedia documents with semantic concepts via Open Calais (http://www.opencalais.com/).

In this work, we present the UI design our multimedia search engine fulgeo. It provides the users both a good overview of the result set as well as intuitive ways to explore the nature of a presentation's content, without the need to fill out complicated forms such as in FLAME [8]. The UI design of our search engine is based on general design recommendations and interviews with three experts.

\section{FULGEO SEARCH UI}

Like in traditional search engines, an initial keyword-based query is entered to fulgeo and matched to the text extracted from the Flash files as well as the semantic concepts associated with the multimedia content. A screenshot of this initial query screen is depicted in Figure 2. The database ranks the results based on their cover density [2]. The engine also supports filtering Flash files for those that contain audio or video files, animations or interaction by clicking on the check-boxes below the query field.

The search result page depicted in Figure 3 provides the users a quick impression of the Flash content by showing a thumbnail for each result. This is a major lack of commercial search engine support for Flash content like Google, which provides only a textual result list. The literature suggests that thumbnails are scanned faster than text and improve the performance of the user to find good results [4]. In order to interactively explore the Flash presentations, we provide intuitive UI features like it 


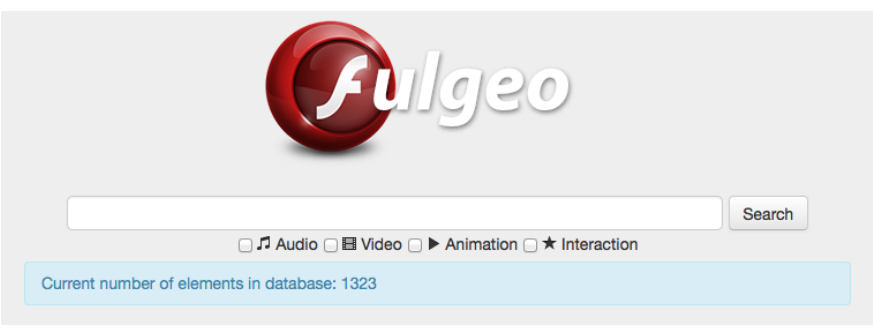

Fig. 2. fulgeo search UI

is known from today's video search engines such as YouTube. For example, we support a mouseover-event of the thumbnail, i. e., a small animation when hovering over a result.

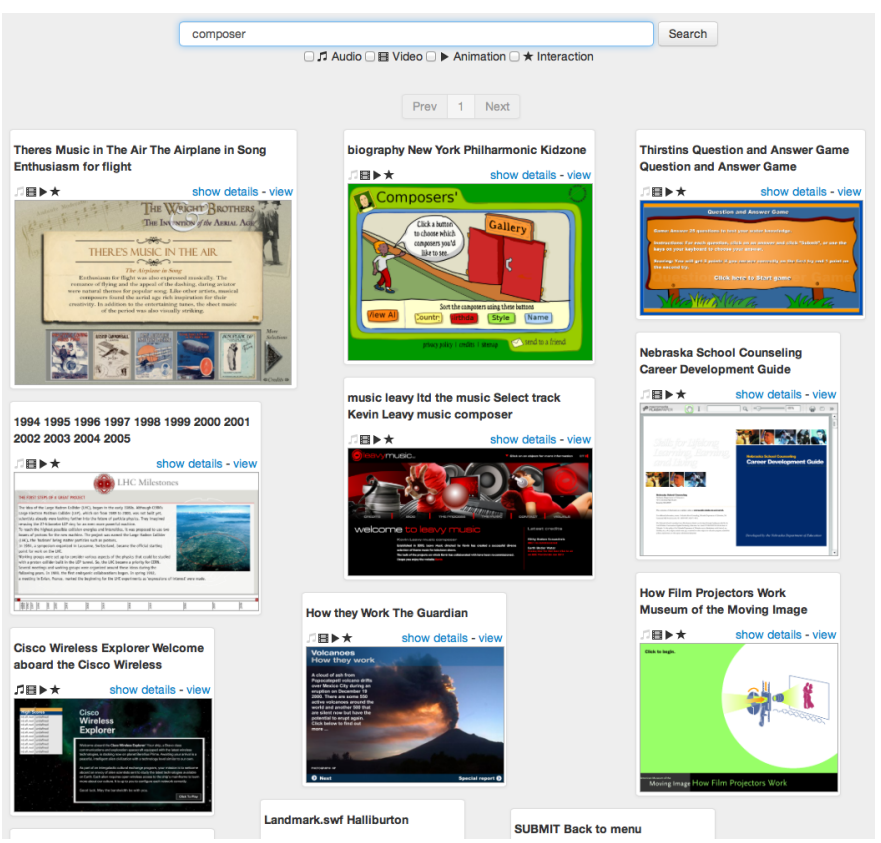

Fig. 3. Results for query 'composer'

To limit the number of results per page, we have introduced a pagination mechanism that splits up the results along sets of 20 items. This seems to be a reasonable compromise without increasing the so-called pogosticking-effect, i.e., users constantly going back and forth in the search result pages (cf. [6], [5]). In addition, the users should get a quick overview whether audio or video content, animations, or interactive parts are included in the Flash documents. This adds important information to the multimedia search results and reduces short term memory load from the user when comparing different results on the same page. Because results with larger thumbnails seem to be more important to the user [6], we have decided to use the thumbnail size as indicator for the absolute relevance of a Flash presentation in context to the user provided query term.

When interested in a particular result, the user can obtain more details of a Flash document by clicking on the thumbnail of that result. In contrast to FLAME, we provide an intuitive depiction of further details of the Flash content together with indicators whether the result contains animations, interactive parts, and others. The detailed view is arranged at the bottom of the screen as shown in Figure 4 and seamlessly integrated with the original result list. This allows for comparing a Flash presentation of interest with the remaining result list. Inspired by the audio-visual media search engine Voxalead (http://voxaleadnews.labs.exalead.com/), the text contained in the document is displayed next to the keyframe to let the user make his own impression of the pertinence of this document. The search terms that appear in the text are highlighted so that the user sees them in their context in the result.

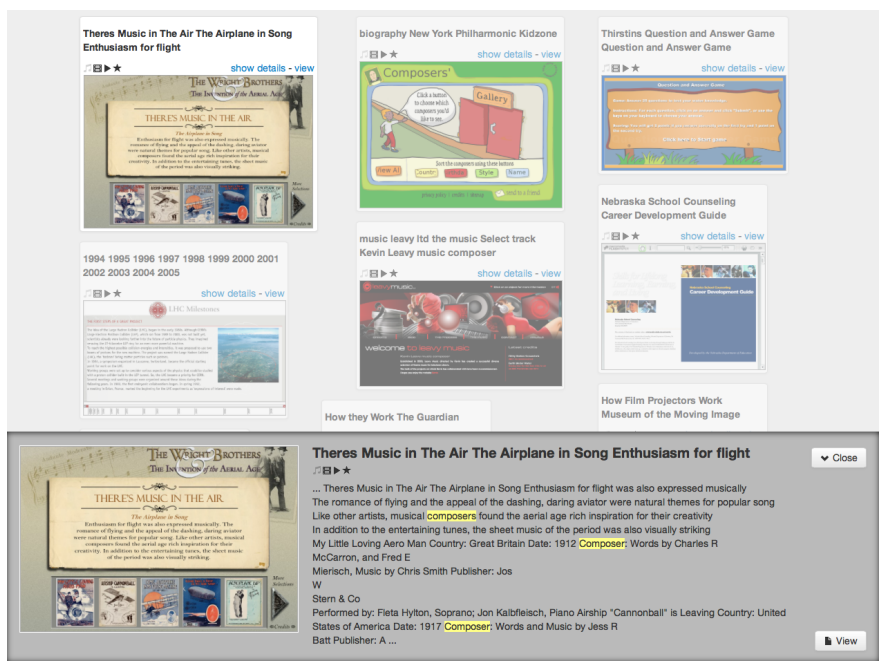

Fig. 4. Details view of search 'composer'

In order to view the document, a link is placed directly next to the thumbnail in the search results page view and details view. Additionally, the document opens when clicking on the big thumbnail inside the view as this is more intuitive than to extra click on a button for that. Finally, the users can also download the presentation.

\section{CONCLUSION}

We presented our prototype fulgeo as a way for a novel user interface for a semantics-enabled multimedia search engine. Currently, the prototype contains more than 4.000 Flash files. As future work, we plan to add at least two orders of magnitude more files. In addition, we plan to conduct extensive user studies.

\section{REFERENCES}

[1] K. S. Candan and M. L. Sapino, Data Management for Multimedia Retrieval. Cambridge Press, 2010.

[2] C. L. A. Clarke, G. V. Cormack, and E. A. Tudhope, "Relevance ranking for one to three term queries," in Computer-Assisted Inform. Retr., 1997.

[3] D. Ding, Q. Li, B. Feng, and L. Wenyin, "A semantic model for flash retrieval using co-occurrence analysis," in Multimedia. ACM, 2003.

[4] M. A. Hearst, Search User Interfaces. Cambridge University Press, 2009.

[5] P. Morville and J. Callender, Search Patterns. O'Reilly, 2010.

[6] G. Nudelman and P. Gabriel-Petit, Designing Search. Wiley, 2011.

[7] L. Weiland and A. Scherp, "A novel approach for semantics-enabled search of multimedia documents on the web," in MMM. Springer, 2014.

[8] J. Yang, Q. Li, L. Wenyin, and Y. Zhuang, "Content-based retrieval of flash movies," Multimedia Tools Appl., vol. 34, no. 1, pp. 1-23, Jul. 2007. 